\title{
The City that We Want: Generative Themes, Constructionist Technologies and School/Social Change
}

\author{
David Cavallo ${ }^{1}$, Paulo Blikstein ${ }^{1}$, Arnan Sipitakiat ${ }^{1}$, Anindita Basu ${ }^{1}$, Alexandra \\ Camargo $^{2}$, Roseli de Deus Lopes ${ }^{2}$, Alice Cavallo ${ }^{3}$ \\ MIT Media Lab ${ }^{1}$, University of São Paulo ${ }^{2}$, Tufts University ${ }^{3}$ \\ [cavallo,arnans,anindia,mello]@media.mit.edu,paulo@northwestern.edu, \\ [roseli,alexiaca]@lsi.usp.br
}

\begin{abstract}
We describe a project, The City that We Want, which enabled the constructionist use of technology within a generative theme to enable students to design and construct their ideas about how to improve life in their communities. We used a variety of computational technologies combined with crafts and scrap materials. The goal was for children to learn in a more contextualized manner important ideas in the disciplines through their projects. We designed the overall project itself as an object to think with in order to facilitate a broader reform in the schools. The willing participation, inspired projects, and commitment and development of the teachers demonstrated significant value.
\end{abstract}

\section{Background}

Implementing real school reform is a difficult proposition. Often the attempted reform does not produce meaningful change. Primary reasons for this are: overcoming existing mindsets of what education has to be; not addressing sufficient number of aspects in a highly connected, complex system; hierarchically instituting a plan designed by others and applied equally without regard to differences in local situation and culture; working only in a pilot effort without realistic models of growth, and not paying attention to implementation $[1,2,3]$.

The difficulty is noticed even though the above list is partial. Yet, it is easy to make the case that developing countries have a rapidly growing need for dramatic improvement in broad public education in order to meet new challenges imposed by the economic changes as we move towards a knowledgebased, creative economy.
Many hold the belief that technology potentially could play an important role in dramatically improving education, in creating the basis for economic growth and more equity, as well as in providing a means for freedom and democracy. We include ourselves among those who hold such beliefs. However, there are many obstacles towards achieving these ideals. Chief among them is the relatively high cost of the technology combined with the lack of people with the experience and expertise to utilize the technology in effective ways.

This paper describes an innovative initiative using technology for learning in municipal public schools in São Paulo, Brazil, and in the national network of freetuition schools run by the Bradesco Foundation. While it is beyond the scope of this paper to provide a full report, we describe some salient results from the project by highlighting certain key cases.

\section{The Challenge}

Upon entering office in 2001 the new mayor of São Paulo appointed Fernando Almeida as the education secretary. In addition to his background in developmental psychology and educational technology, Almeida also worked closely with Paulo Freire and deeply understands principles of pedagogy of liberation and critical thinking [4]. Our research group met with Almeida early in his administration and discussed possible interventions to improve the quality of education and to use technology in innovative ways to improve the learning environment.

At the initial meeting Almeida insisted that we must do something that can impact the entire system. He emphasized that there were more than one million students in the municipal system, and we must propose something that could benefit all. 


\section{A Cidade que a Gente Quer}

D. Cavallo proposed a project called A Cidade Que a Gente Quer, which translates more or less as The City that We Want. In the project the students design and construct models of how they would like to improve the quality of life in their city. The idea was an extension of work performed in the United States and Thailand $[5,6]$. The goal of the project is to create a rich learning environment of interest to the participants where they build computational models to express their ideas. The basic premise is that students will perform a critical inquiry into the life, culture, and functioning of their city and create new models of how they would like some aspects to be. They can address something they perceive as problematic (e.g. waste recycling, transportation, energy use, crime, etc.), or propose a model of a dream, providing something desired but not previously possible (e.g. dynamic customizable clean transportation, instant playgrounds, responsive environments, etc.).

The project builds upon three lines of thinking about learning; the constructivism of Piaget [7], the critical consciousness through engagement with one's environment of Freire [8], and the constructionism of Papert [9]. Constructionism builds upon the Piagetian constructivist idea that learners construct their own knowledge by asserting that this process happens especially felicitously through the thoughtful and reflective design and construction of personally meaningful artifacts [9]. The unifying concept of the city provides a Freirean generative theme whereby any participant will have their own ideas and beliefs and can use these to guide their conception and implementation. Moreover, the participants can identify with the situation as they can place themselves inside their projects, using this as a means to guide their design.

We also wanted the project to better re-link the schools and their communities. The community provides the basis for study and content. The school becomes a contributor to the improvement of its host community. Moreover, we designed the project so that students could place their projects on the web and to discuss the merits of the ideas and analysis through a forum. This would provide the basis for discourse among students regarding their conceptions of problems, causes, and solutions. A major goal in addition to the learning in mathematics, science, and other disciplines, was for the students to develop the belief that they could have impact on their environment and that they could be agents for positive change. Just as the desire to make a design implementation actually function forces the learner to deal with the underlying scientific principles, the desire to convince other participants of one's own analysis and solution requires the learner to support his or her argument with data and reasoning. Unfortunately, the planned connectivity was not achieved during the initial phases of our project.

The project should serve as an object to think with [10]. That is, as we wanted this to contribute to a longer term reform of the educational environment, we proposed the project as a concrete example of how to work in a more open, active learning environment and what content could be different. We would utilize this experience as a case for the teachers and administrators to reflect upon ideas for teaching and learning. The project is case-based active learning at the macro, systemic level.

Our project-based approach differs from other project-based implementations through the use of generative themes. Rather than specifying the exact project and its steps, this is open for the students to decide. This aspect is critical in several respects. First, it engages the learners in a critical engagement with their community. Second, it places the student in the active agency of determining methodology such that they develop this capability for approaching future problems. Third, it subsumes the problems embedded within the successful completion of the project. Thus, our approach connects to problem-based approaches of using real-world problems as objects of study [11], but contextualizes the problems within the projects.

\section{Materials}

We used a variety of materials. In order to animate the model, they used the programmable GoGo board, designed to enable real-time interaction between outside devices and computer programs. The design was also optimized not for highest and latest functionality, but for low cost, locally available parts, and human scale for assembly [12].

We introduced another design consideration to lower costs by asking everyone to bring in scrap materials, broken electronics, mechanical devices; whatever material that could be scavenged for parts. Our original conception was that this would lower costs. However, something much more interesting and important happened! Another empowering element emerged as familiar objects whose mechanism was opaque and unfamiliar to most everyone became understandable and usable. When our participants took them apart they saw how they worked, and then found ways to adapt those mechanisms into useful 
apparatuses for their own projects. Moreover, this reappropriation of materials fit within the culture of students who came from humble households.

\section{Preparation}

We did not want to impose our project fully designed from the top down. Our desire for development and sustainability led us to take an emergent participatory design approach [6]. We do not believe that forcing teachers to participate will achieve good results. Thus we only worked with schools and teachers that volunteered to participate. In order to give the educators a chance to decide, we composed a document to explain the ideas, principles and approach, as well as to suggest sample projects. We then ran a vision seminar to present and discuss the concepts and to demonstrate the technologies and possible projects. We only had enough staff to handle fifty schools in the first semester plus around forty more from Bradesco, although more volunteered than we could manage properly.

We did not dictate how a school must adopt the program. They could choose to run it during school hours or afterwards and with whichever combination of teachers and subjects they desired. We chose to take a participatory approach so as to gain their fuller energies, to take advantage of their local knowledge of communities, schools, and the children (and thus avoiding a major pitfall of standardized, top-down, hierarchical approaches), and to prepare for local sustainability.

We ran initial workshops at Poli lab at the University of São Paulo (USP) for the technology division of the secretariat and the technology support team. The site itself made an important contribution as the teachers knew that USP had a world-class engineering program, but did not know how it operated. By working in the labs, and having the opportunity to observe an engineering challenge in which first-year university students were competing and where the challenge was similar to what we were doing with them, it strengthened their views of themselves and dispelled their doubts about whether our approach was merely playing or was serious and beneficial. We ran the next phase in a few schools, with the previous groups combined with teachers and students from the schools. We asked the teachers to accompany us to the electronics district of São Paulo to purchase materials. We asked them to videotape the process to document the work and create materials for subsequent groups. We did not believe that these materials would be sufficient for others to learn the process, ideas, and tools. Rather, we believed that by learners taking responsibility for the documentation and explaining the ideas to others, the process would prove useful as a learning device for the documenting group. The idea was to learn by documenting, not to learn merely through reading documentation.

\section{Projects}

It is not possible here to describe all the projects. However, the range and sophistication of projects was incredible. Moreover, the spirit and diligence demonstrated by the students in and of itself made the whole endeavor worthwhile. We present a few representative samples to highlight certain aspects to the work. Still, we are compelled to point out that many projects were truly works of inspiration and creativity. There were streetlights powered by the movement of the cars on the street; devices to detect and remove trash from rivers; many devices to aid the handicapped such as a steering device for the paraplegic, movable wheelchair ramps, and responsive transit; automatic recycling devices; playgrounds for slum areas; devices to measure water usage and reappropriate it; devices for sewage disposal and water purification; a school turned into an art exhibit of the wall of problems and tree of solutions with the students' projects the linkage from problem to solution; and even an answer robot that would respond with the students' solutions to the posed problems.

In one school in the north zone of São Paulo, in a tough neighborhood with high economic needs, a group of students proposed the idea that violence in the neighborhood and in homes is increased because their parents had to take long uncomfortable bus rides for hours in each direction in order to get to work. The students believed that this experience increased tension, which fostered aggressive behavior. They proposed a new bus, to have air conditioning, music, and display if the bus was full so that people at the bus stops would know why it would not stop. When we asked how would the bus "know" it was full, they said the seats could all have sensors. When we asked what other device might operate this way, immediately one of them said "A keyboard!" We took a broken one apart and they saw its mechanism was just a number of spatially located electronic contacts. In their messing about with the robotic materials, they became familiar with this mechanism. They then wired their keyboard bus to red and green LEDs, a speaker from a radio, and a fan from broken power supply (figure 1). What is particularly important about this project is that these youth who worked on this project did not perform well 
in school at all. They barely even attended! Their teacher told us that if you asked them a question, you would only receive a blank stare as a response. Within this project, even though they were only scheduled to attend morning sessions of the school, they remained throughout the day and into the evening working on their projects, only leaving when we requested so that we too could leave for dinner.

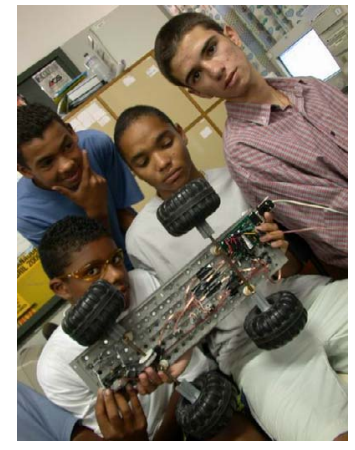

Figure 1- Intelligent Bus

Another salient example was from students in Bodoquena, a residential school situated in the world's largest wetlands. As part of larger project examining water use issues, the students discovered that the greatest use of water at school was the 100,000 liters of water drained from the school's swimming pool each week. They traced its path, tested the water quality at a variety of stations, and used their data to build a simulation model of how they could treat this water and use it to create a fish farm. They developed a mathematical model and proved to the school that it was a feasible and profitable project. The foundation had wanted a fish farm in order to teach the children the skills so that they could create fish farms for their families to improve their livelihood. However, the foundation had rejected the idea as too costly. The students' innovative application and proof of safety enabled its creation and on-going operation.

The youngest original participants were from a second-grade classroom, also in a poor section of the north zone of São Paulo. For a group of girls, the biggest problem in São Paulo was for them to go to the bathroom in the school. It was dirty, graffiti-filled, had no toilet paper, and they were often harassed by boys. Their idea was to create "The Bathroom that We Want." They created a photo-journalist essay on the travails of going to the bathroom. When the deputy mayor saw their project, he committed to paying more attention to the school bathrooms in his jurisdiction.

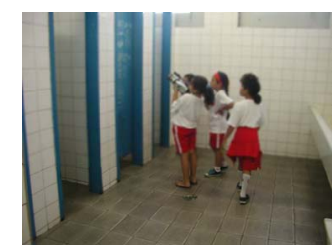

Figure 2-Investigative journalists

This project in particular highlighted an interesting phenomenon. The 8-year-old girls were able to articulate a problem in a sophisticated (and effective!) manner that they could not have done with text. We typically ask students to keep design notebooks to document their projects. The vast majority of students resist this approach. In this project we had digital still and video cameras, and asked them to document their work through image, text, video, and voice annotation. Every group did this! Moreover, the sophistication and level of articulation was truly high. They are fluent with creating and understanding using image even though hardly any had ever composed with video and image before. As a tool for reflection, broadening the palette of tools had a remarkably positive effect. In addition, their documentation is providing us with an important database of project images, mechanisms and explanations that is helping us to overcome obstacles to growth, both by enabling technical learning and this different pedagogical approach.

\section{Discussion}

The bottom-up request for continuity of the project is perhaps the best evidence of one aspect of its effectiveness. It is non-trivial to conceive, design, and implement such authentic projects, and often harder than much of the typically assigned classwork. Yet students and teachers willingly spent extra time in order to participate. The science, mathematics, civic studies, and communication achievement was remarkable, and did not fit within the past performance of many of the participants.

Learning in this approach is very much a social experience. People share ideas, the less experienced learn from the more experienced, ideas are discussed and tested, people switch back and forth between the roles of master and apprentice, teacher and student, expert and novice.

By involving learners in real engineering and design work, our learning projects give learners direct experience with deliberative democracy. Through open discussions people pick meaningful problems to work on, propose and evaluate design ideas, consider tradeoffs, and arrive at workable solutions. This emergent paradigm for learning provides a way to reconnect 
with the community, particularly for alienated and marginalized young people. It also provides an experience for them to be effective change agents within their communities.

Working in this different learning paradigm enables people to build up confidence in their own ability to learn new things, their ability to develop new skills, and their ability to acquire the new knowledge they may need to achieve their goals. For people previously marginalized by their failure in the standard schools or by lack of opportunity for formal education, this combined boost in self-confidence and tangible skills can lead to reawakening hope.

The experience with one of the district-level technical support teachers provides an interesting glimpse into issues of success and failure. Initially she was uncomfortable with our way of working. To her, the open-ended learning strategy of our different paradigm seemed disorganized.

By the end of the project, this woman had become our most vocal supporter. How did this come about? She had experienced a transformation in her own learning and her own confidence in herself as a learner.

Previously all her technical knowledge was of a narrow procedural kind. She admitted that she had been afraid of technology, afraid to venture off the known path and explore on her own. Before taking part in the learning project, she had never taken anything apart before in her life. Watching how naturally the young students got into it, she said she "went running after them" knowing she could do it too. She saw how much the students could do. She had the spirit to try to do it herself, to try to learn, to learn how to do new things with the technology.

She got the fundamental message of the new paradigm: We may not already know all we need to know in advance, but we can learn as we go along designing and building and testing out ideas.

\section{Limitations}

In addition to the lack of resources and the delays in equipment delivery and connectivity, the project faced significant turmoil due to a lack of organizational continuity. In the course of three years we worked with three different secretaries. The city re-organized the support mechanism, moving from 13 districts to 31 . In the schools teachers would often leave their jobs or change to other schools. In order for any reform to be effective, it needs time for implementation. If there is constant change and turmoil, no reform can take root.
Still, despite difficulties, the research team remains in admiration of the teachers and administrators who continue to function and devote tremendous energy and passion towards the education of the children in their charge despite low pay and often times poor treatment.

\section{Next Steps}

We are grateful that in both systems are continuing the project autonomously. The systems have expanded the reach to more schools. Indeed, due to the roots in Piaget, Freire, and constructionism, other places in Brazil as well as in Costa Rica with similar traditions, have adopted and implemented the ideas. As they have become more fluent with the materials and ideas, the projects are going deeper. We are working to help develop more facilitators, and provide more technological tools, better support materials, and new content and generative themes [13].

\section{References}

[1] D. Tyack and L. Cuban, Tinkering Towards Utopia: A Century of Public School Reform, Harvard University Press, Cambridge, Ma., 1995

[2] S. Papert, "Why School Reform is Impossible", Journal of the Learning Sciences, 6(4), pp. 417-427, 1997.

[3] D. Cavallo, Models of growth, forthcoming.

[4] P. Freire, Pedagogy of the Oppressed, Herder and Herder, New York, 1972.

[5] D. Cavallo, "New Initiatives in Youth Development: Technology Works Enterprises," in Proceedings of ICLS 96, AACE, 1996, pp. 9-13.

[6] D. Cavallo, "Emergent Design and learning environments: Building upon indigenous knowledge", IBM Systems Journal, vol. 39, nos. 3-4, 2000, pp. 768-781.

[7] H. E. Gruber \& J. J. Voneche, eds., The Essential Piaget, Basic Books, New York, 1977.

[8] P. Freire, Pedagogy of the Oppressed, Herder and Herder, New York, 1972.

[9] S. Papert, "Introduction", in Constructionism, S. Papert and I. Harel editors, Ablex, Norwood, NJ, 1991.

[10] S. Papert, Mindstorms: Children, Computers and Powerful Ideas, Basic Books, New York, 1980.

[11] J. Rhem, Problem-Based Learning: An Introduction, in National Teaching and Learning Forum, vol.8 \#1, 1988.

[12] A. Sipitakiat, P. Blikstein, and D. Cavallo, "The GoGo Board: Moving towards highly available computational tools in learning environments", Proceedings of Interactive Computer Aided Learning International Workshop. Carinthia Technology Institute, Villach, Austria, 2002.

[13] D. Cavallo, et. al., "RoBallet", International Conference for the Learning Sciences, Los Angeles, Ca., 2004. 